\title{
Research Progress on Acupuncture Treatment of Knee Osteoarthritis
}

\author{
Zhang-Yueyu ${ }^{1}$, Song- Aiqun ${ }^{2}$ and Peng Ying ${ }^{3}$ \\ ${ }^{1}$ College of Acupuncture, Moxibustion and Orthopedics, Hubei University of Traditional Chinese Medicine, Wuhan City, Hubei \\ Province, China \\ ${ }^{2}$ Department of Acupuncture and Moxibustion, Hubei Provincial Hospital of Traditional Chinese Medicine, Wuhan City, Hubei \\ Province, China \\ ${ }^{3}$ College of Acupuncture, Moxibustion and Orthopedics, Hubei University of Traditional Chinese Medicine, Wuhan City, Hubei \\ Province, China
}

\section{Asian Journal of Complementary and Alternative Medicine. Volume 09 Issue 4}

Published on: 22 /11/2021

${ }^{*}$ Author for Correspondence: Song- Aiqun, Department of Acupuncture and Moxibustion, Hubei Provincial Hospital of Traditional Chinese Medicine, Wuhan City, Hubei Province, China; E-mail: 245855503@qq.com

Cite this article as: Yueyu Z, Aiqun S, Ying P. Research Progress on Acupuncture Treatment of Knee Osteoarthritis, Vol 9(4), 106110:2021.

\begin{abstract}
Osteoarthritis of the knee is a common clinical degenerative disease, which occurs in middle-aged and elderly people. Acupuncture therapy has a significant effect in the treatment of knee osteoarthritis, and is gradually accepted by more and more patients. Observing the treatment of knee osteoarthritis by different acupuncture and moxibustion therapies, we can find that acupuncture treatment of knee osteoarthritis has the advantages of relieving knee joint pain, improving knee joint function, absorbing knee joint inflammation, and promoting the recovery of damaged soft tissue. The treatment of osteoarthritis provides a more effective treatment plan.
\end{abstract}

Keywords: Acupuncture therapy; Knee joint; Osteoarthritis

\section{INTRODUCTION}

Knee osteoarthritis is a joint degenerative disease, the most common in osteoarthritis, the main symptoms are knee pain, joint stiffness, restricted mobility, and even seriously affect the patient's quality of life [1]. This disease mostly occurs in middle-aged and elderly people, but also in young people. It can be seen in unilateral knee joints, and can also be seen in bilateral disease. And the prevalence of women is higher than that of men [2]. With the development of aging society, the incidence of the disease is gradually increasing, so it is increasingly important to optimize the treatment plan of KOA. Modern medicine often adopts the "anti-inflammatory + analgesic + cartilage protection" treatment mode in the treatment of knee osteoarthritis. Oral western medicine has unstable efficacy and many adverse reactions, especially for people with kidneys, liver, and stomach and duodenum. In KOA patients with diseases and middle-aged and elderly KOA patients, severe gastrointestinal reactions and liver and kidney function damage will bring more negative effects to patients [3]. Acupuncture is one of the external treatments of traditional Chinese medicine. It has been affirmed by domestic and foreign medicine in the treatment of knee osteoarthritis. Compared with oral western medicine treatment and surgical treatment, it is also economical, safe, simple and easy to implement. Unique advantages such as fewer adverse reactions [4]. It has been accepted by a large number of patients, and the methods of acupuncture and moxibustion treatment of KOA are becoming more and more diversified.

\section{UNDERSTANDING OF KNEE JOINT OSTEOARTHRITIS IN TRADITIONAL CHINESE MEDICINE}

Knee osteoarthritis belongs to the category of "bone arthritis" in Chinese medicine [5]. "Suwen-Bilun" put forward that "the three qi of wind, cold and dampness are mixed, and they become arthralgia." The concept of "Muscle Bi", "Pibi" and "Gubi" Wutibi. Knee osteoarthritis belongs to "Gubi" in "Wubi". Knee osteoarthritis is divided into 3 syndrome types in the "Diagnosis and Efficacy Criteria for Diseases and Syndromes of Traditional Chinese Medicine": liver and kidney deficiency type, spleen and kidney yang deficiency type, and phlegm and blood stasis block type [6]. Modern medical research on KOA syndrome types mostly focuses on the distribution of 3 or 4 syndrome types [7]. Research by Li Gubao et al. [8] found that the overall syndromes of KOA can be divided into deficiency syndromes, positivity syndromes, and deficiency-excess syndromes. The deficiency syndromes are mainly liver and kidney deficiency, kidney deficiency and marrow deficiency, 
and spleen and kidney deficiency. The main methods are tonic: Nourishing liver and kidney, nourishing spleen and kidney, nourishing kidney and filling marrow. The study found that the overall syndromes of KOA can be divided into deficiency syndromes, positive syndromes, and deficiency and excess syndromes. The empirical evidence includes cold-dampness obstruction type, qi stagnation and blood stasis type, damp-heat congestion type, tendons and vein stasis type, and rheumatism obstruction type. To remove blood stasis and collaterals, expel wind and dampness. The syndrome of deficiency and excess is mainly based on yang deficiency and cold coagulation type, qi deficiency and dampness resistance type, and the treatment principle is to warm the middle to nourish the yang and promote qi and dehumidification. Acupuncture therapy, as a kind of external treatment of Chinese medicine, is also closely related to KOA. In the chapter of "Lingshu·Hailun": "The meridians belong to the internal organs, and the external collaterals are in the limbs", which shows that the meridian is very close to the joints, muscles and bones. In the "Lingshu Benzang" chapter: "The meridian can promote blood and yin and yang, and the muscles and bones can help the joints", which also shows that the meridian points have the effect of treating muscles, bones and joints [9].

\section{TREATMENT METHOD}

Common acupuncture and moxibustion therapies at present mainly include filiform needle therapy, fire needle therapy, electroacupuncture therapy, warm needle moxibustion therapy, acupuncture knife therapy, moxibustion therapy, comprehensive therapy, etc.

\section{Filiform Needle Therapy}

Fibre-needle therapy, also known as body acupuncture, is a method of acupuncture through the meridians and acupoints of the human body using fili-needles as acupuncture tools. It is also the most commonly used acupuncture therapy in clinical practice.Qiu Rui et al. [10] used acupuncture to treat 70 patients with knee arthritis. The acupuncture group was given Knee Eye, Liangqiu, Yanglingquan, and Kneeyangguan as the main points, while the control group was given sodium hyaluronate injection and glucosamine. The results show that acupuncture is better than sodium hyaluronate injection and oral glucosamine in alleviating the pain symptoms of KOA and the analgesic effect. Acupuncture can promote blood circulation, improve the stability of the knee joint, and relieve the pain of the knee joint. After acupuncture, the index of knee joint flexor and extensor muscle strength is significantly improved. Wu Weibin et al. [11] used Qi-acupuncture to treat 72 patients with knee osteoarthritis. The results showed that Qi-acupuncture was better than simple acupuncture in terms of reducing VAS pain degree score and WOMAC score, indicating that Qiacupuncture treatment of wind, cold and dampness KOA is effective and feasible. Compared with ordinary acupuncture, Qi-acupuncture has a more obvious therapeutic effect on KOA. Especially in the small and deep parts of the treatment, Qi-acupuncture can be used to strengthen stimulation. The role of feeling and enhancing the sense of qi.

\section{Fire Needle Therapy}

Fire needle therapy is a method in which special needles are burned red and quickly inserted into acupoints or affected areas to treat diseases [12]. Wang Bing et al. [13] used fire needle-raising acupuncture to treat KOA compared with filineedle acupuncture. The two groups of acupuncture points were selected as Xuehai, Liangqiu, Kneeyan, Tubi, Zusanli, Yanglingquan, and Yinlingquan, and found that fire-needle raising Acupuncture has a higher rate of cure for $\mathrm{KOA}$, a faster effect time, and a more significant analgesic effect. The curative effect is also better than that of simple filiform acupuncture. Tang Zhigang et al. [14] chose to use fire acupuncture for the study of knee arthritis, fire acupuncture treatment as the observation group, filigree needle treatment as the control group, the severity index score of the observation group was significantly lower than that of the control group. It can be seen that fire needle therapy also has a significant effect in the treatment of KOA, and has the effects of repairing soft tissue damage, anti-inflammatory and pain relief, and can improve the blood flow state and microcirculation of the knee joint.

\section{Electroacupuncture Therapy}

Electroacupuncture therapy is a method in which the pulse current output by the electroacupuncture device is applied to the meridian acupoints through milli-needles. Guo Yanming et al. [15] used electro-acupuncture to treat early and mid-term KOA to observe the effect of electro-acupuncture. The total effective rates of electro-acupuncture group and western medicine group were $88.9 \%$ and $80 \%$, respectively. It can effectively improve patients' joint pain, stiffness, and joint mobility, but the longterm effects need to be further observed. Gang Jiahong et al. compared the clinical efficacy of electroacupuncture and meloxicam for early and mid-term KOA and found that both electroacupuncture and oral meloxicam are effective in early and mid-term KOA treatment, but the curative effect of electroacupuncture is much better than that of oral meloxicam [16]. By improving the muscle tension of the rectus femoris muscle, electroacupuncture can help restore the internal mechanical balance of the knee joint, thereby improving knee joint function. This shows that electroacupuncture has great advantages in the early and mid-term KOA treatment and is worthy of promotion in clinical treatment. 


\section{Warm Needling Therapy}

Warm needle therapy is a therapy that uses the warm moxibustion sensation produced by the burning of moxa to act on the acupoints or affected areas after the acupuncture has gained qi. The combined application of acupuncture and moxibustion. Wei Xiangdong [17] uses warm needle moxibustion to treat senile KOA. Warm needle moxibustion can improve local blood circulation, enhance the patient's immunity, and promote the recovery of knee joint function. It can also promote the relief of joint pain and delay the deformation of bones and joints. Tu Xuesong et al. [18] used warm needling and sodium hyaluronate intra-articular injection as a control. After half a year of treatment, the Lequesne score and VAS score of the warm needling moxibustion group were lower than those of the control group. It can be seen that the curative effect of warm needling moxibustion on KOA is definite. The dual effect can effectively improve and relieve knee pain in patients, and the long-term effect (half a year after treatment) is significant.

\section{Acupotomy Therapy}

Acupotomy therapy is a kind of closed relaxation therapy, which is a new type of minimally invasive surgery. It is to pierce acupotomy into the deep part of the treatment point to loosen and peel the lesion to achieve the purpose of soothing the meridian and relieving the collaterals [19]. Guo Changqing et al. [20] used acupotomy to relieve the pain of knee osteoarthritis, and treated 60 patients with KOA with longitudinal dredge and transverse dissection with acupotomy. The results showed that acupotomy can relieve the pain of knee osteoarthritis patients well. Symptoms and pain relief are better than electroacupuncture, and there are no adverse reactions and events, suggesting that acupotomy is an effective and safe treatment for KOA. Wang Xiang et al. [21] treated 115 patients with KOA with acupotomy meridian layer relaxation. After 4 weeks of treatment, the scores of the acupotomy group were better than those of the acupuncture group in terms of pain symptoms, knee squatting, long standing pain, and improvement in daily activities. Acupotomy therapy is superior to acupuncture therapy in terms of relieving overall pain symptoms and restoring joint mobility, but the effects of both are equivalent in terms of stiffness and walking pain. Although it can be concluded from the research results that the overall effect of acupotomy for KOA is better than that of acupuncture, as a new type of technology, acupotomy transmuscular layer release requires strict adherence to safe and effective operations during the operation. It can guarantee its safety and effectiveness.

\section{Moxibustion Therapy}

Moxibustion mainly uses the physical and chemical effects of the burning of moxa sticks to promote the circulation of local Qi and blood through the channels along the meridian, so as to achieve the purpose of curing diseases. "The needle is inaccessible, the moxibustion does" shows that moxibustion is widely used and can be used in the treatment of KOA. Yuan Qingdong et al. [22] found that the effect of thunder fire-heatsensitive moxibustion on KOA was significantly better than oral diclofenac sodium enteric-coated tablets. The medicinal factors produced by the burning of a variety of traditional Chinese medicines in thunder fire moxibustion penetrate into the tissue cells through heat radiation to circulate in the tissues and cells. It promotes the material exchange of tissue cells, so as to exert the efficacy of drugs, and combine with the sensitizing effect of heat-sensitive moxibustion to achieve the effect of treating KOA. Nie Bin et al. [23] used Fuyang fire moxibustion to treat knee osteoarthritis of yang deficiency and cold coagulation type, and found that Fuyang fire moxibustion can achieve a good analgesic effect and significantly improve the symptoms of yang deficiency. Fuyang fire moxibustion combines the advantages of moxibustion, transdermal drug delivery of traditional Chinese medicine, and acupoint stimulation, and can be promoted as a better clinical treatment method. Compared with acupuncture therapy, moxibustion therapy can be easier to operate, and patients who have a sense of fear and resistance to acupuncture can use moxibustion therapy.

\section{Comprehensive Therapy}

Comprehensive therapy is a compound therapy, and the methods of comprehensive treatment are also different. Generally, acupuncture and moxibustion are combined with other treatment methods to treat knee osteoarthritis, and its clinical treatment effect is greatly improved than that of single therapy. Weng Xianming et al. [24] used ear acupoint pressing combined with wrist-ankle acupuncture and cupping to treat KOA, exerting that ear-point pressing can relieve pain, wrist and ankle acupuncture can relieve pain, and cupping can dredge the meridians. Compared with a single treatment method, the multi-modal combined analgesia treatment of KOA has better curative effect. Li Shilin et al. [25] used electroacupuncture combined with traditional Chinese medicine fumigation to treat KOA, and the results showed that the therapy of electroacupuncture combined with traditional Chinese medicine fumigation was more significant than that of oral non-steroidal anti-inflammatory drugs. By leveraging the advantages of acupuncture, traditional Chinese medicine, and fumigation physiotherapy, combining the three can 
significantly relieve knee joint pain, improve patients' clinical symptoms, improve knee muscle endurance, promote the absorption of intra-articular inflammation, and repair damaged tissues, thereby achieving the purpose of treating KOA. It can be seen that comprehensive treatment of KOA can exert its synergistic effect, thereby improving the overall treatment effect.

\section{DISCUSSION}

Knee osteoarthritis is a degenerative joint disease with complex pathogenesis, which may be related to factors such as obesity, age, family inheritance, inflammation, trauma, etc. [26]. The pathological features are mainly manifested as articular cartilage destruction, subchondral bone sclerosis, and joint bone hyperplasia [27]. Studies [28] have shown that traditional Chinese medicine has a definite effect in treating KOA patients in the early and mid-term. It can relieve knee joint swelling and pain, and improve knee joint function. Acupuncture, as a kind of external treatment of traditional Chinese medicine, has obvious effects and advantages in the treatment of knee osteoarthritis, and the methods of acupuncture treatment of KOA are becoming more and more diversified. At the same time, the evaluation criteria of curative effect after KOA treatment are also gradually increasing, these all show that acupuncture treatment KOA has been universally recognized and widely used.

At present, acupuncture and moxibustion treatment of KOA mainly adopts comprehensive therapies, and a single treatment method is rarely used, and all doctors believe that the efficacy of comprehensive therapy for knee osteoarthritis is better than that of single therapy, but how should the various treatment methods be coordinated? Application can achieve the best clinical effect, and further exploration and research are still needed. At the same time, the research of acupuncture and moxibustion treatment of KOA also has the following shortcomings: 1. Lack of complete and objective curative effect evaluation criteria; 2. Unclear classification of TCM syndrome types for KOA; 3. Lack of evidence-based medicine evidence and theoretical support; 4. KOA pathological mechanism is complicated. The mechanism of action of different treatment methods needs to be further improved. Therefore, when studying acupuncture and moxibustion treatment of KOA in the future, it is necessary to formulate more complete efficacy evaluation standards, and carry out higher-quality clinical randomized controlled trials to determine the specific syndrome types of KOA treatment, and conduct differentiation and treatment according to different syndrome types to make the treatment more accurate [29]. Physicians should pay more attention to changes in symptoms before and after treatment, objective inspection indicators, subjective scale evaluation, and consistency of intervention methods within the group. At the same time, follow-up work is carried out to observe the long-term efficacy of patients and record adverse reactions. Through further exploration of the research progress of acupuncture and moxibustion treatment of KOA, we hope to provide a more accurate and effective plan for clinical treatment of KOA.

\section{REFERENCES}

1. Wang B, Nansheng Y (2019) Expert consensus on step treatment of knee osteoarthritis. Chinese Journal of Joint Surgery 13: 124130 .

2. Tang X, Shengfeng W, Siyan Z, Niu J, Zhang Y, et al. (2016) The Prevalence of Symptomatic Knee Osteoarthritis in China: Results from the China Health and Retirement Longitudinal Study. Arthritis \& Rheumatology 68: 648-653.

3. Likun S, Xunjie Z, Zhiyan L, Guo DY, Duan YW, et al. (2018) Evaluation of the efficacy of 11 interventions in the treatment of knee osteoarthritis. Tianjin Traditional Chinese Medicine 35: 116-121.

4. Zhenyuan M, Hui M (2020) Research progress in the treatment of knee osteoarthritis with traditional Chinese medicine application. Journal of Nanjing University of Traditional Chinese Medicine 36: $926-930$.

5. Yingrui M, Yuqian Z (2019) Understanding and Research on Knee Osteoarthritis in Traditional Chinese Medicine. Clinical Research of Traditional Chinese Medicine 11: 146-148.

6. Shaofeng C, Yunzhu L, Ruizhi L (2019) Overview of clinical research on acupuncture treatment of knee osteoarthritis. Hunan Journal of Traditional Chinese Medicine 35: 183-185.

7. Xiaofeng Q, Tengteng W, Qianqian L (2016) Research status of traditional Chinese medicine syndromes of knee osteoarthritis. World Science and Technology-Modernization of Traditional Chinese Medicine 18: 1879-1882.

8. Gubao L, Lei Z, Shangke Q (2014) Analysis of the efficacy of oral prescriptions and TCM syndrome types in the literature of knee osteoarthritis in the past 10 years. China Journal of Orthopaedics and Traumatology 22: 20-21.

9. Shuai Y, Feng X, Lina Q (2016) Literature review of acupuncture treatment of knee osteoarthritis in the past 5 years. World Journal of Integrated Traditional Chinese and Western Medicine 11: 278-280.

10. Rui Q, Yuan Z, Wenbao W (2020) Acupuncture treatment of 34 cases of knee osteoarthritis. Fujian Traditional Chinese Medicine 51: $22-24$.

11. Weibin W, Qun F, Fan W (2018) Clinical study on Qi-acupuncture treatment of knee osteoarthritis of wind-cold-dampness-type arthritis. China Emergency in Traditional Chinese Medicine 27: 1137-1139.

12. Zhong W (2015) Treatment of 39 Cases of Knee Osteoarthritis with Fire Needle Combined with Chinese Medicine Oral Administration. Journal of Traditional Chinese Medicine Research 28: 45-47. 
13. Bing W, Jing H, Ning Z (2017) Clinical Observation on the Treatment of Knee Osteoarthritis with Fire Needle and Yang Acupuncture. Chinese Acupuncture and Moxibustion 37: 463-466.

14. Zhigang $T$ (2014) The clinical efficacy of fire needling in the treatment of knee arthritis. Guide to Chinese Medicine 12: 294-295.

15. Yanming G, Yongying L, Junqing G (2016) Treatment of early and mid-stage knee osteoarthritis with electroacupuncture. Jilin Journal of Traditional Chinese Medicine 36: 91-93.

16. Jiahong G, Yiqun M, Huamin W (2016) Comparison of the clinical efficacy of electroacupuncture and meloxicam in the treatment of early and mid-stage knee osteoarthritis: A randomized controlled study. Chinese Journal of Acupuncture and Moxibustion 36: 467470.

17. Xiangdong W (2018) Clinical Observation on Treatment of Senile Knee Osteoarthropathy with Warming Needle Moxibustion. Guangming Traditional Chinese Medicine 33: 3549-3551.

18. Xuesong T, Xiaoan L, Lixia H (2016) A clinical randomized controlled study of warm needling moxibustion in the treatment of knee osteoarthritis. Journal of Clinical Acupuncture and Moxibustion 32: 38-40.

19. Minglei Z, Yuehong B, Ying Z (2017) Research progress on the treatment of knee osteoarthritis with a small needle knife. Hebei Journal of Traditional Chinese Medicine 39: 1908-1912.

20. Changqing G, Tong S, Jianmin W (2012) A randomized controlled clinical study of acupotomy to improve knee osteoarthritis pain symptoms. Tianjin Journal of Traditional Chinese Medicine 29: 35-38.

21. Xiang W, Shunyi L, Ying S (2016) Clinical observation of acupotomy in the treatment of knee osteoarthritis. China Orthopedics and Traumatology 29: 345-349.
22. Qingdong Y, Xin G, Yacen H (2015) Observation on the curative effect of thunder fire-heat-sensitive moxibustion on knee osteoarthritis. Shanghai Journal of Acupuncture and Moxibustion 34: 665-668.

23. Bin N, Yuan T, Zhengping S (2017) Clinical observation of Fuyang fire moxibustion in treating knee osteoarthritis of yang deficiency and cold coagulation. Rheumatism and Arthritis 6: 2225 .

24. Xianming W, Xiaoli C, Jiefeng H (2017) Ear point pressing combined with wrist-ankle acupuncture and medicine cup in the treatment of mild to moderate knee osteoarthritis. Chinese Medicine Orthopedics 29: 51-54.

25. Shilin L, Min A, Yan X (2018) Observation on the efficacy of electroacupuncture combined with traditional Chinese medicine fumigation in the treatment of knee osteoarthritis. Hebei Traditional Chinese Medicine 40: 109-112.

26. Weiheng C (2020) Guidelines for Diagnosis and Treatment of Knee Osteoarthritis in Traditional Chinese Medicine. TCM Bone Surgery $32: 1-14$.

27. McGrory B, Kristy W, John AL, John CR, Moore DC, et al. (2016) The American Academy of Orthopaedic Surgeons EvidenceBased Clinical Practice Guideline on Surgical Management of Osteoarthritis of the Knee. The Journal of bone and joint surgery. American volume 98: 688-692.

28. Lele H, Lei C, Wenxiang G (2016) Current status of treatment of knee osteoarthritis with traditional Chinese medicine. Rheumatism and Arthritis 5: 77-80.

29. Ruoxu W, Yanxing G, Jiayi G (2019) Research progress in internal treatment of knee osteoarthritis with traditional Chinese medicine. Electronic Journal of Clinical Medicine 6: 195-198. 